\title{
Micro-computed tomography study of the abnormal osseous extensions of sella turcica
}

\author{
J. Skrzat ${ }^{1}$, M. Kozerska ${ }^{1}$, A. Wróbel ${ }^{2}$ \\ ${ }^{1}$ Department of Anatomy, Jagiellonian University, Collegium Medicum, Cracow, Poland \\ 2Department of Medical Physics, Institute of Physics, Jagiellonian University, Cracow, Poland
}

[Received 20 June 2013; Accepted 14 August 2013]

\begin{abstract}
The paper presents anatomical considerations on the abnormal ossification, which occurred around the dorsum of the sella turcica in the human skull of the female individual. Probably the morphological alterations of the sellar region were associated with extensive heterotopic ossification of the dura mater attached to the dorsum of the sella turcica and the posterior clinoid processes. The analysis of gray values of the voxels representing the areas of abnormal ossification indicated on variation in bone density in the entire sample. We have established that the highest mineralisation of bony tissue occurred in the marginal parts of the osseous extensions deriving from the posterior clinoid processes. The ossified parts of dura mater attached to the posterior clinoid processes showed significantly higher content of the hydroxyapatite $\left(1.9 \mathrm{~g} / \mathrm{cm}^{3}\right)$ than the dorsum of the sella turcica $\left(1.0 \mathrm{~g} / \mathrm{cm}^{3}\right.$ ). (Folia Morphol 2014; 73, 1: 19-23)
\end{abstract}

Key words: sella turcica, heterotopic ossification, bone mineralisation

\section{INTRODUCTION}

The bands of the dura mater that extend between the osseous components of the middle cranial fossa may ossify occasionally. In consequence, various bony bridges may be formed between different parts of the sphenoid bone and adhering bones (e.g. petrous bone). The ossified dural bands usually resemble ribbons, trabeculas or spikes. In most cases incomplete or complete bony bridges are formed. Effects of heterotopic bone formation within the interclinoid ligaments, caroticoclinoid, and petroclinoid ligaments are widely described in literature. They can cause clinical effects or remain asymptomatic $[3,4,10,11]$.

The aim of this study was to examine the microstructure of the abnormal osseous formations of the uncommon shape, which arise posteriorly from the dorsum sellae and the posterior clionoid processes of the sphenoid bone.

\section{MATERIALS AND METHODS}

During visual inspection of the cranial collection that is housed in the Department of Anatomy of the Collegium Medicum of the Jagiellonian University, a skull of the adult female individual revealed unusual osseous formations of the dorsum of the sella turcica. The skull was dissected horizontally, and the calvaria was removed to show the effect of abnormal ossification of the sellar region (Fig. 1). The external appearance of the abnormal osseous formations of the dorsum sellae was documented by the photography using digital camera (Canon EOS 5D), equipped with the Tamron Macro lens.

Further, anomalous bony formation together with the dorsum of the sella turcica was isolated from the body of the sphenoid bone and subjected to the microtomographic examination. The osseous sample was scanned with a Skyscan 1172 microtomograph

Address for correspondence: Dr J. Skrzat, Department of Anatomy, Jagiellonian University, Collegium Medicum, ul. Kopernika 12, 31-034 Kraków, Poland, tel: +48 1242295 11, fax: +48 1243030 06, e-mail: jskrzat@poczta.onet.pl 


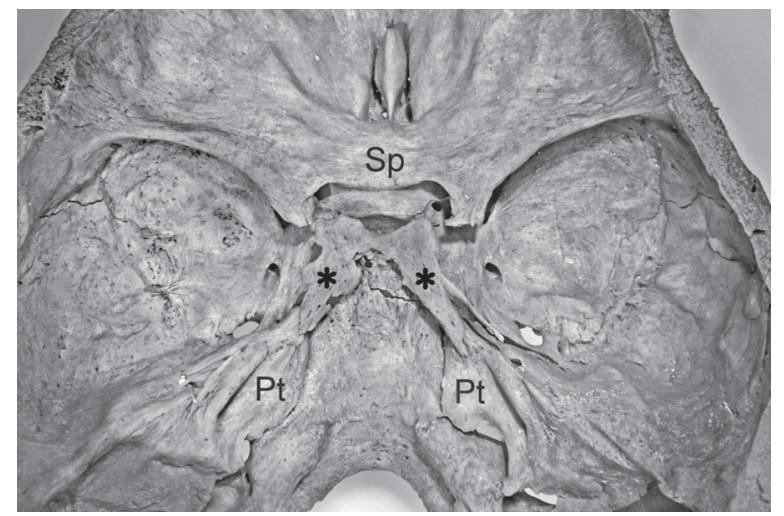

Figure 1. Internal view of the cranial base showing anomalous bony formations of the dorsum sellae (marked by the asterisks); $\mathrm{Sp}$ - sphenoid bone, $\mathrm{Pt}$ - petrous bone.

(Skyscan, N.V., Aartselaar, Belgium). The scanner was equipped with the $\mathrm{X}$-ray detector: 11 Megapixel (4024 $\times 2680$ in total; $4000 \times 2400$ effective), 12-bit digital X-ray camera with $24 \times 36 \mathrm{~mm}$ field of view. The X-ray source voltage was set to $80 \mathrm{kV}$ and current to $100 \mu \mathrm{A}$. The image pixel size was $13.7 \mu \mathrm{m}$. The projection images were acquired over an angular range of $180^{\circ}$ with an angular step of $0.5^{\circ}$. The interval between the slices that make up the volume was $13.6 \mu \mathrm{m}$. The projections were reconstructed using a cone-beam reconstruction software (NRECON ver. 1.6.5 SkyScan), based on the Feldkamp algorithm.

Visualisation of the osseous structure and the air spaces was performed in the CTVox SkyScan software (ver. 2.3), which allows performing multi-planar reconstruction and volume rendering of the image data. Additionally, the channels of luminance and opacity (opposite of transparency) were tuned up by changing transfer function which governs the way of mapping in the image. By modifying the opacity we could control the visibility of the corresponding voxels and thus enhance imaging of the air spaces in the sella turcica.

Radiographic imaging of the sellar region included 2-dimensional cross-sectional views and the 3-dimensional reconstruction. This procedure helped to establish anatomic configuration of the bony tissue within the ossified parts of the dura mater. The compact bone from the cancellous bone was differentiated based on the texture analysis of the image, and the level of mineralisation was depicted by variation of gray shades. Gray values (0-255) of the voxels reflected bone density in the sample. In this tonal range, black is represented as 0 and white colour is denoted by
255. According to this notation, brighter areas within the image correspond with higher density of tissue within the sample. To quantify the intensity of pixels in the image, we performed a computer image analysis of the selected computed tomography (CT) scans taken from distinct parts of the sample of the ossified tissue. This procedure served to create a map of the areas showing the highest mineralisation. For this purpose we used free public domain image processing software "ImageJ" (http://imagej.nih.gov/ij) [1] In turn, the degree of mineralisation expressed by hydroxyapatite content ( $\mathrm{g}$ mineral $/ \mathrm{cm}^{3}$ ) was calculated using the computer software CT-Analyser, ver. 1.11, which enables deriving quantitative parameters from scanned datasets obtained with SkyScan microtomograph. Calibration of the dataset was performed by means of phantoms with known bone mineral density defined as the volumetric density of calcium hydroxyapatite (CaHA) expressed in $\mathrm{g} / \mathrm{cm}^{3}$ [2].

\section{RESULTS}

Visual inspection of the basicranium revealed a peculiar form of anomalous ossification that occurred in the area of the dorsum sellae. In the examined skull, the right and the left posterior clinoid process are well developed. They project from the dorsum sellae in the anterolateral direction, but their superolateral corners are extensively overgrown. The corners of the dorsum sellae and both posterior clinoid processes are considerable widened and stretched in the posterolateral direction. The accessory osseous formations of the dorsum sellae resemble butterfly wings or can be termed as the wings of the dorsum sellae. Bone overgrowth of the dorsum sellae was imagined by the micro-CT scans (Fig. 2).

A remarkable feature of the examined sample is the presence of numerous apertures or clefts that penetrate osseous extensions of the right and left posterior clinoid process. All these apertures are very narrow and orientated along the axis of both osseous extensions of the posterior clinoid processes. Most of them perforate completely the bone (Fig. 2). The occurrence of these apertures is a side effect of interlacing bony trabeculas, which compose the extensions of the dorsum sellae and the posterior clinoid processes.

It was noted in the micro-CT scans that dorsum sellae is well pneumatised. The middle part of the dorsum sellae was slightly eroded and in the mico-CT scans it appeared as a translucent area. This indicates 

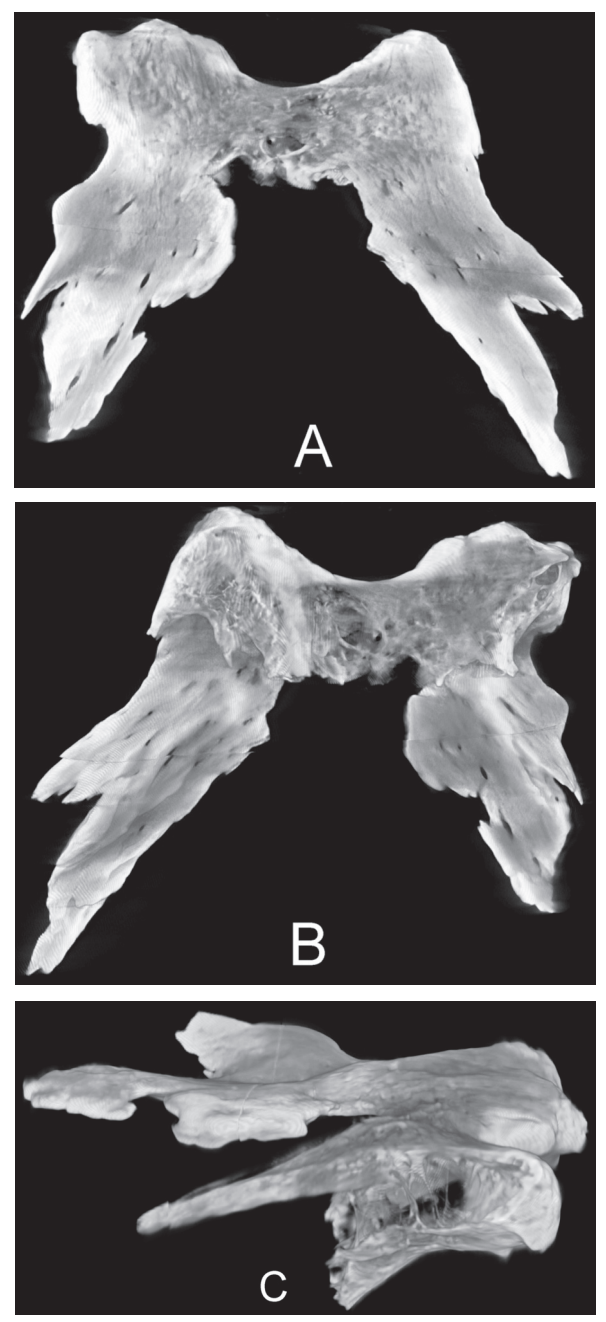

Figure 2. A volume rendering image obtained from the micro-computed tomography of the dorsum sellae turcica with adjacent abnormal osseous structures combining the posterior clinoid processes; A. Superior view; B. Inferior view; C. Lateral view.

on vivid decline of the inner table of the compact bone, and the loss of bone density.

We have also found out that the dorsum sellae contains the areolar space, which extends laterally towards the posterior clinoid processes. Inside these osseous components, small air chambers were detected. The biggest chambers were observed within the corns of the posterior clinoid processes. Posterior parts of the posterior clinoid processes were divided into irregular compartments filled with the air. The septation of the air chambers of the dorsum sellae and both clinoid processes was made of bony trabeculas, which are orientated vertically and obliquely (Fig. 3).

Sequential transverse micro-CT scanning of the ossifications that extended backwards from the

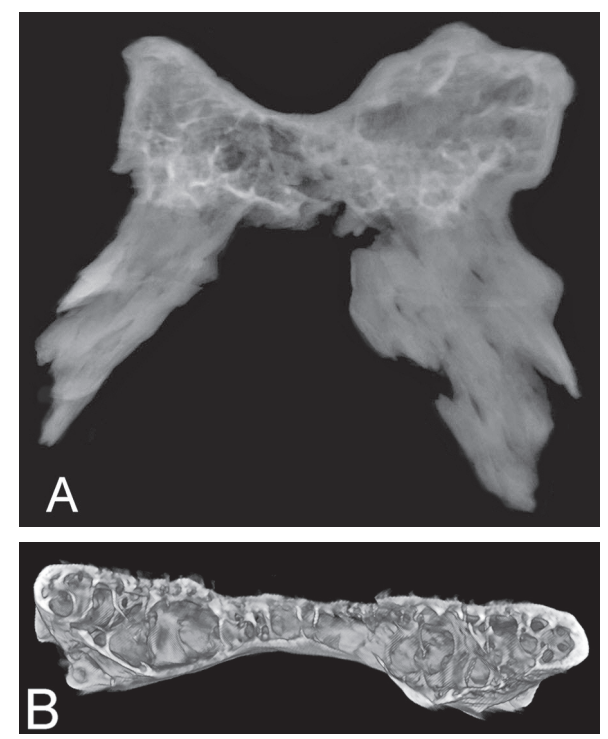

Figure 3. A radiograph of the dorsum sellae $(\mathbf{A})$ and the 3-dimensional reconstruction of the air cells arrangement (B) showing extensive pneumatisation.

dorsum sellae showed alteration of the osseous composition. In these osseous components the spongy bone disappeared, and the compact bone, which was well mineralised, dominated. However, the compact substance was not homogenous in the whole area of the heterotopic ossification. Texture analysis of the micro-CT scans revealed the presence of numerous brighter circular spots, which were aggregated mostly along the peripheral layer of the ossified dura mater. In the transverse section it resembles tiny granulations that adhere to each other, forming a dense osseous cortex. In fact, these are bony trabeculas, which lay down in the matrix of the dura mater during its heterotopic ossification. The trabeculas were arranged in longitudinal manner, from the top of the posterior clinoid process they spread mainly posteriorly, probably along the bundles of the collagen fibers, which gradually calcified. Coronal CT scans demonstrated spots of bony tissue condensation within abnormal extensions of the sellar region. In fact, these spots are transverse sections through the aggregates of the osseous trabeculas, which laid down in the dura mater and caused its ossification. Longitudinal arrangement of the bony trabeculas can be perceived on the inferior aspect of the specimen and is also visible as darker spots in the transverse section (Fig. 4).

In Figure 4B, the plot profile displays a 2-dimensional graph of the intensities of pixels along a line within the image. The $x$-axis represents a distance 


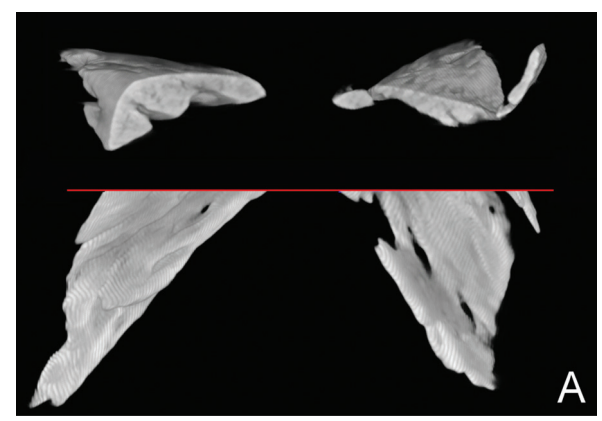

B

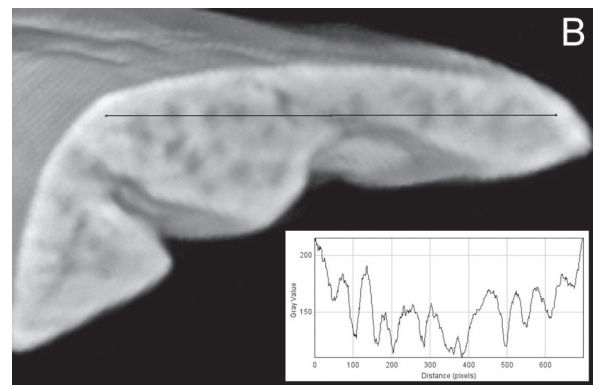

Figure 4. The transverse section of the anomalous osseous extensions of the sellar region (A); Close-up view on the transverse section of the left ossified sellar extension (B); the graph indicates on inhomogeneous osseous matrix assessed along the profile line.

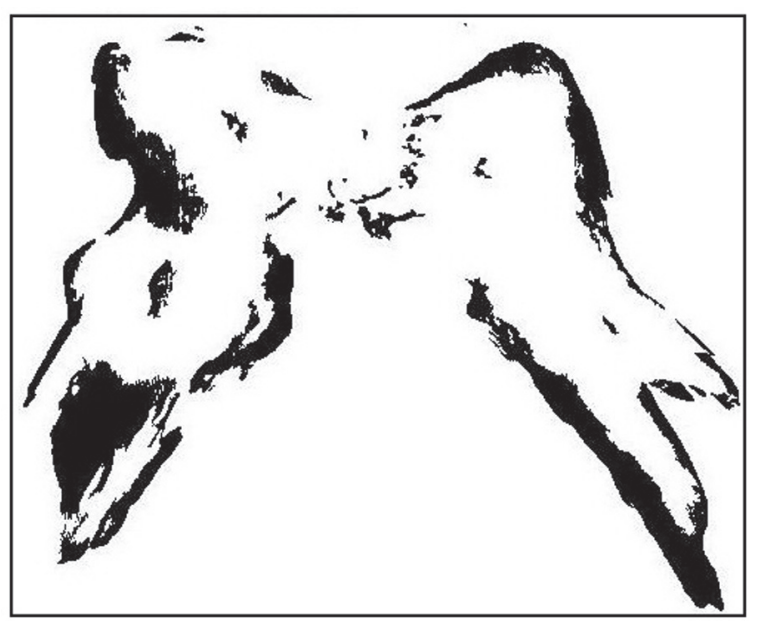

Figure 5. Map of the areas of the extensive mineralisation (marked by the black colour).

along the line and the $y$-axis is the pixel intensity. It is well visible that pixels intensity changes considerably in approximate similar intervals. This fluctuation refers to reciprocal changes of bone density caused by non-uniform mineralisation. Thus, the bony matrix of the ossified extension of the sellar region shows inhomogeneous condensation of the bony disuse. Local changes of the bone mieral density were depicted in Figure 5. This image was obtained as maximum intensity projection applied to the sample

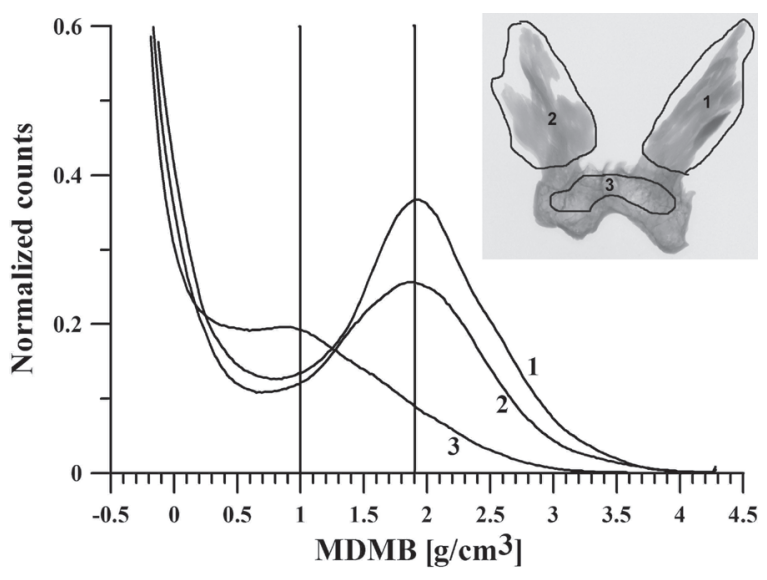

Figure 6. Mean degree of mineralisation of bone (MDMB) in 3 regions of the examined sample. The parts of the sample (1 and 2) resulted from heterotopic ossification show significantly higher content of the hydroxyapatite $\left(1.9 \mathrm{~g} / \mathrm{cm}^{3}\right)$ than the dorsum sellae $\left(1.0 \mathrm{~g} / \mathrm{cm}^{3}\right)$ marked as region 3 , which originates from normal ossification.

examined in the micro-CT. In this case, only voxels with the highest attenuation value are visible in the image and they just represent the areas of the highest mineralisation.

We have concluded that strong mineralisation occurred in the entire sample, but dominated in the posterior part of the bony extensions of the dorsum sellae. The regions of highest mineralisation were located along the edges of the extensions of the posterior clinoid processes. The extensive mineralisation occurred along the medial edge of the left and the right osseous extension, resembling wings. Also, the middle part of the left wing was strongly mineralised (Fig. 5). The result of the image analysis supports measured content of the hydroxyapatite, which expresses the mean degree of bone mineralisation (Fig. 6).

\section{DISCUSSION}

The dorsum sellae is usually a square projection of bone ended with 2 eminences called the posterior clinoid processes located behind the sella turcica. Normal anatomy of the sella turcica and adjacent osseous components can be altered by disturbances in development of the sphenoid bone $[8,12]$. Dietemann et al. [5] described a sellar spine which arose in the midline of the anterior aspect of the dorsum sellae and was directed towards the centre of the sella turcica. This abnormality can be congenital, but it is also regarded as calcified notochordal remnants, ossified dural fold or, ossified vascular channel. 
In the studied case, abnormal ossification appeared as expanding mass of bony tissue, leading to posterior elongation and widening of the posterior clinoid processes. The posterior clinoid processes are overgrown by the osseous tissue, which could result from heterotopic ossification of the dura mater attached to the sellar region. Morphological appearance and shape of the sellar formation suggests that these are ossified dural bands, that were originally stretched between the posterior clinoid processes and the petrous apex of the temporal bones. Their position corresponds with the course of the petrosphenoid ligament, connecting the lateral margin of the dorsum sellae to the border of the petrous bone [9]. Another ossified entity arising from the posterior clinoid processes towards the petrous apex seems to be the ossified petroclinoid ligament, which is normally composed of the dens fibrous connective tissue [13].

The dura mater may undergo local heterotopic ossification or calcification, which extend vary across the individuals, depending on the ethiopathologic factors. Vidya et al. [14] reported on unilateral calcification of the petrosphenoid ligament, whereas other researchers found a complete petrosphenoid bony bridge both in male and female skulls $[4,10]$. Also the petroclinoid ligament may calcify, but this condition is usually regarded as the age-related degeneration having laminar or nodular pattern [7].

Current study demonstrates a vivid case of ossification that occurred within the dural bands anchored to the dorsum sellae. The process of ossification must have been extensive, because it manifested in formation of large, wing-shaped structures located bilaterally and spread widely in the postero-lateral direction. We have not found in the available literature any description of similar form of ossified dura mater of the dorsum sella. This ossification concerns apparently the petrosphenoid and petroclinoid ligaments, converting them into the bony lamellas or spikes.

In the studied case, the reason of ossification of the sellar region is not established. Such abnormal condition might have been dictated by hormonal disorder or accompanied metabolic disease. Some authors mention that formation of the osseous abnormalities within the sphenoid bone may have endocrinological background. Stanton and Wilkinson [11] found familial calcification of the petrosphenoid ligaments, which took the form of hook directed backwards and downwards from the posterior clinoid process. The calcification of ligamentous structures was also seen as the continuous line running from the posterior clinoid process to the apex of the petrous bone. Lang noted that calcification of the petroclinoid ligament is considered to be an anatomic anomaly related to basal cell carcinoma syndrome and systemic fluorosis [8].

\section{CONCLUSIONS}

In our case, the ossification around the dorsum sellae supported by strong mineralisation of the bony structure suggests that it might have been a congenital anomaly [6, 7]. Certainly, it was a long-term process related with a gradual calcification and bone tissue formation within the bands of the dura mater inserted to the dorsum sellae.

\section{ACKNOWLEDGEMENTS}

The research was carried out with the equipment purchased thanks to the financial support of the European Regional Development Fund in the framework of the Polish Innovation Economy Operational Program (contract no. POIG.02.01.00-12-023/08).

The study was conducted with the approval of the Bioethics Committee of the Jagiellonian University (KBET/109/B/2012).

\section{REFERENCES}

1. Abràmoff MD, Magalhães PJ, Ram SJ (2004) Image Processing with Image J. Biophotonics Int, 11: 36-42.

2. Boivin G, Meunier PJ (2002) The degree of mineralization of bone tissue measured by computerized quantitative contact microradiography. Calcif Tissue Int, 70: 503-511.

3. Callender JS (1995) Non-progressive familial idiopathic intracranial calcification: a family report. J Neurol Neurosurg Psychiatry, 59: 432-434.

4. Cederberg RA, Benson BW, Nunn M, English JD (2003) Calcification of the interclinoid and petroclinoid ligaments of sella turcica: a radiographic study of the prevalence. Orthod Craniofac Res, 6: 227-232.

5. Dietemann JL, Bonneville JF, Cattin F, Poulignot D (1983) Computed tomography of the sellar spine. Neuroradiology, 24: 173-174.

6. Kendall B, Cavanagh N (1986) Intracranial calcification in pediatric computed tomography. Neuroradiol, 28: 324-330.

7. Kıroğlu Y, Callı C, Karabulut N, Öncel C (2009) Intracranial calcifications on CT. Diagn Interv Radiol, 16: 263-269.

8. Lang J (1977) Structure and postnatal organization of heretofore uninvestigated and infrequent ossifications of the sella turcica region. Acta Anat, 99: 121-139.

9. Liu X, Xu Q, Che X, Mao R (2009) Anatomy of the petrosphenoidal and petrolingual ligaments at the petrous apex. Clin Anat 22: 302-306.

10. Peker T, Anil A, Gulekon N, Turgut HB, Pelin C, Karakose M (2006) The incidence and types of sella and sphenopetrous bridges. Neurosurg Rev, 29: 219-223.

11. Stanton JB, Wilkinson M (1949) Familial calcification of the petrosphenoidal ligament. Lancet, 2: 736-740.

12. Sirikci A, Bayazit YA, Bayram M, Mumbuc S, Gungor K, Kanlikama M (2000) Variations of sphenoid and related structures. Eur Radiol, 10: 844-848.

13. Skrzat J, Walocha J, Jaworek JK, Mróz I (2007) The clinical significance of the petroclinoid ligament. Folia Morphol, 66: 39-43.

14. Vidya CS, Shamasundar NM, Saraswathi G (2012) A case report of unilateral calcification of petrosphenoid ligament. Anat Karnataka, 6: 17-19. 